\title{
GEOMETRIC MEANS AND HADAMARD PRODUCTS
}

\author{
BAO Qi Feng AND Andrew TONGE
}

\begin{abstract}
Ando [1] proved that for $m$ commuting positive definite matrices, the $m$-fold Hadamard product of their geometric mean is bounded above by their Hadamard product. We obtain a natural extension to the non-commutative case.
\end{abstract} product.

Mathematics subject classification (2000): 15A24, 15A45, 15A48, 15A69, 47A63, 47A64.

Key words and phrases: arithmetic mean, geometric mean, $\alpha$-power mean, Hadamard product, tensor

\section{REFERENCES}

[1] T. ANDO, Concavity of certain maps on positive definite matrices and applications to Hadamard products, Linear Algebra Appl., 26, (1979), 203-241, MR 80f: 15023.

[2] R. Bhatia, Matrix analysis, Springer-Verlag New York, 1997, MR 98i: 15003.

[3] B. Q. FenG, A. M. TONGE, Matrix versions of some refinements of the arithmetic-geometric mean inequality, submitted.

[4] R. A. Horn, C. R. Johnson, Matrix Analysis, Cambridge University Press 1990, MR 91i:15027.

[5] W. Pusz, S. L. Woronowicz, Functional calculus for sesquilinear forms and the purification map, Reports on Mathematical Physics, 8, (1975), 159-170, MR 54: \# 8316.

[6] M. SAGAE, K. TANABE, Upper and lower bounds for the arithmetic-geometric-harmonic means of positive definite matrices, Linear and Multilinear Algebra, 37, (1994), 279-282, MR 95m: 15030. 